\title{
Inorganic nanoparticles kill Toxoplasma gondii via changes in redox status and mitochondrial membrane potential
}

This article was published in the following Dove Press journal:

International Journal of Nanomedicine

28 February 2017

Number of times this article has been viewed

\author{
Oluyomi Stephen \\ Adeyemi ${ }^{1,2}$ \\ Yuho Murata' \\ Tatsuki Sugi' \\ Kentaro Kato' \\ 'National Research Center for \\ Protozoan Diseases, Obihiro \\ University of Agriculture and \\ Veterinary Medicine, Obihiro, \\ Japan; ${ }^{2}$ Medicinal Biochemistry and \\ Toxicology Laboratory, Department \\ of Biological Sciences, Landmark \\ University, Omu-Aran, Nigeria
}

Correspondence: Kentaro Kato National Research Center for Protozoan Diseases, Obihiro University of Agriculture and Veterinary Medicine, Inada-cho, Obihiro, Hokkaido 080-8555, Japan

Tel +8I I55 495645

Fax $+8 I I 55495646$

Email kkato@obihiro.ac.jp
Abstract: This study evaluated the anti-Toxoplasma gondii potential of gold, silver, and platinum nanoparticles (NPs). Inorganic NPs $(0.01-1,000 \mu \mathrm{g} / \mathrm{mL})$ were screened for antiparasitic activity. The NPs caused $>90 \%$ inhibition of $T$. gondii growth with $\mathrm{EC}_{50}$ values of $\leq 7, \leq 1$, and $\leq 100 \mu \mathrm{g} / \mathrm{mL}$ for gold, silver, and platinum NPs, respectively. The NPs showed no host cell cytotoxicity at the effective anti- $T$. gondii concentrations; the estimated selectivity index revealed a $\geq 20$-fold activity toward the parasite versus the host cell. The anti- $T$. gondii activity of the NPs, which may be linked to redox signaling, affected the parasite mitochondrial membrane potential and parasite invasion, replication, recovery, and infectivity potential. Our results demonstrated the antiparasitic potential of NPs. The findings support the further exploration of NPs as a possible source of alternative and effective anti- $T$. gondii agents.

Keywords: antiparasite, drug screening, nanomedicine, toxoplasmosis

\section{Introduction}

Toxoplasma gondii is an intracellular parasite belonging to the Apicomplexa family. ${ }^{1}$ T. gondii is the causative agent of toxoplasmosis, which is one of the most common parasitic diseases. ${ }^{2}$ Toxoplasmosis is common because the parasite has low host specificity and therefore can infect a range of hosts, including nearly one-third of the human population. ${ }^{3}$ Toxoplasma infection can be asymptomatic in healthy individuals but fatal in pregnant or immunocompromised individuals; it has been linked to abortion, fetal abnormalities, and encephalitis in these individuals. ${ }^{4}$ In healthy individuals who are infected with Toxoplasma, the multiplication of the parasites is handled by the immune system and the administered drugs, but cysts remain in all infected tissues, including the brain, thereby serving as a source for exacerbations. Therefore, efficient treatment of all infected cells to prevent cyst formation is imperative.

Current treatment options for toxoplasmosis patients are limited. They include the use of antimalarial drugs or antibiotics, which often cause significant side effects, including bone marrow suppression and rashes. ${ }^{4}$ Current therapy for toxoplasmosis suppresses the active infection but does not cure the latent infection and is poorly tolerated. Without prolonged suppressive treatment, up to $80 \%$ of cases relapse, and $20 \%-30 \%$ of patients on suppressive therapy relapse. The side effects of drugs have led to discontinuation of therapy in up to $40 \%$ of patients. ${ }^{3,5}$ Consequently, toxoplasmosis represents a large global burden that is further enhanced by the shortcomings of the current therapeutic options. These factors underscore the urgent need for better anti-Toxoplasma drugs and/or new approaches in the treatment of toxoplasmosis. 
An ideal anti-Toxoplasma drug would be potent, nontoxic, and able to eliminate latent infection.

There is expanding interest in deploying nanotechnology for biomedical purposes, ${ }^{6}$ and some reports suggest that nanoparticles (NPs) could form the bulk of future treatment strategies for various diseases. ${ }^{7,8} \mathrm{NPs}$ are already being exploited for biomedical applications, due to their nanoscale size and other remarkable properties, including surface reactivity. ${ }^{9}$ These particles can produce reactive oxygen species (ROS) that have the ability to kill infectious agents. ${ }^{10,11}$ Furthermore, the small size of NPs allows them to transverse membrane barriers, leading to greater reactivity. ${ }^{12}$ In addition, NPs could be accumulated in tissues,${ }^{13}$ thereby presenting a formidable platform to target $T$. gondii cysts in host tissues. Of special interest are the metal NPs such as gold and silver, which have antimicrobial activity, ${ }^{14,15}$ antiparasitic activity, ${ }^{16,17}$ and other bioactivities, including the selective inhibition of some enzyme activities. ${ }^{918-21}$ The versatility of metal $\mathrm{NPs}^{10}$ makes them an attractive choice to be explored further as antiparasitic agents, particularly against toxoplasmosis. Here, we explored the potential of metal NPs as novel anti- $T$. gondii agents using in vitro experimental infection models.

\section{Materials and methods Materials}

Inorganic NPs, including gold NPs (AuNPs; $5 \mathrm{~nm}$ ), silver NPs (AgNPs; $10 \mathrm{~nm}$ ), and platinum NPs (PtNPs; $3 \mathrm{~nm}$ ), were purchased from (Sigma-Aldrich, St Louis, MO, USA). The NPs were used as supplied following characterization to confirm the supplier's specification. The NPs were reconstituted in fresh culture medium prior to each use. All reagents were of analytical grade and used as supplied unless otherwise stated.

\section{In vitro anti-T. gondii potential of metal NPs \\ Parasites}

The $T$. gondii $\mathrm{RH}$ strain $2 \mathrm{~F}^{22}$ was used for this study unless otherwise stated. The parasite was maintained by repeated passages in monolayers of human foreskin fibroblast (HFF) cells (American Type Culture Collection, Manassas, VA USA) cultured in Dulbecco's Modified Eagle's medium (DMEM; Nissui, Tokyo, Japan) supplemented with GlutaMAX TM_I (Thermo Fisher Scientific Inc, St Louis, MO, USA), $10 \%$ (v/v) fetal calf serum (FCS; Gibco), and penicillin and streptomycin (100 U/mL; Thermo Fisher Scientific Inc). Host cells infected with $T$. gondii tachyzoites were passed through a $27 \mathrm{G}$ needle to lyse them. The cell lysates were then filtered through a $5 \mu \mathrm{m}$ filter to obtain a tachyzoite suspension free of host cell debris. The suspension was washed with fresh culture medium. Then, the parasite density was measured using a hemocytometer and adjusted for in vitro experimental infection analysis.

\section{In vitro invasion and growth inhibition assessment by luciferase reporter assays}

The number of $T$. gondii tachyzoites was determined using a luminescence-based assay of $\beta$-galactosidase ( $\beta$-gal) activity expressed by the parasite strain RH-2F. To obtain a purified parasite suspension for the assays, infected cells were syringe released and the lysates were filtered to remove cell debris.

First, the anti-T. gondii $\mathrm{EC}_{50}$ and $\mathrm{IC}_{50}$ values for host cell toxicity were determined. In this preliminary assay, the NPs (AuNPs, AgNPs, and PtNPs) were screened at various concentrations ranging from 0.01 to $1,000 \mu \mathrm{g} / \mathrm{mL}$. Based on the preliminary results, further experimental screening was done using concentrations of 15,10 , and $1,000 \mu \mathrm{g} / \mathrm{mL}$ for AuNPs, AgNPs, and PtNPs, respectively.

The growth inhibition assays and in vitro invasion assays were performed as described by Ishiwa et al. ${ }^{22}$ For the growth inhibition assay, purified parasite suspension was added to growing monolayers and invasion was allowed to occur for $1 \mathrm{~h}$. The medium was then removed, the monolayers were washed thoroughly, and fresh medium containing the NP solutions (reconstituted in culture medium) was added. The monolayers were then incubated for $48 \mathrm{~h}$. For the invasion assay, purified parasite suspension and the NP solutions (AuNPs, AgNPs, and PtNPs freshly reconstituted in culture medium) were added to the growing monolayers and incubated for $1 \mathrm{~h}$ to allow for invasion. The medium was then removed, the monolayers were washed thoroughly to remove uninvading parasites, and fresh medium was added. The monolayers were then incubated for $48 \mathrm{~h}$. To assay invasion and growth inhibition, purified parasite suspension and the NP solutions (reconstituted in culture medium) were added to the growing monolayers and incubated for $48 \mathrm{~h}$. The mock-treated (treatment with NP vehicle; in this case, culture medium) cells served as a positive control, whereas the medium-only well was used to correct for any background signal noise. After $48 \mathrm{~h}$ incubation at $37^{\circ} \mathrm{C}$ in a $5 \% \mathrm{CO}_{2}$ atmosphere, the viability of the $\mathrm{RH}-2 \mathrm{~F}$ parasite strain was determined by assaying for galactosidase expression using a Beta-Glo ${ }^{\circledR}$ Luminescent Assay kit (Promega, Madison, WI, USA). The assay was performed in triplicate and repeated three times. All experiments were performed in 96-well optical bottom plates (Nunc; Fisher Scientific, Pittsburgh, PA, USA) unless otherwise stated. 


\section{Host cell viability}

\section{Cytotoxicity of metal NPs in mammalian cells}

HFF cells were maintained in DMEM supplemented with GlutaMAX ${ }^{\mathrm{TM}}-\mathrm{I}, 10 \%$ (v/v) FCS, and penicillin and streptomycin $(100 \mathrm{U} / \mathrm{mL})$. Cells were grown to confluence at $37^{\circ} \mathrm{C}$ in a $5 \% \mathrm{CO}_{2}$ atmosphere. All experiments were performed in 96-well plates (Nunc) unless otherwise stated. At confluence, cells were trypsinized and resuspended to the desired cell density. The cells were seeded onto plates at a density of $1 \times 10^{5}$ cells/well and incubated for $72 \mathrm{~h}$ followed by treatment with various concentrations (between 0.01 and $1,000 \mu \mathrm{g} / \mathrm{mL}$ ) of the NPs (AuNPs, AgNPs, and PtNPs). Culture medium lacking the test compounds was added to the control well, and the medium only well was used to correct for any background signal noise. The treated cells were incubated for $72 \mathrm{~h}$ before being subjected to the cell viability assay. All experiments were performed in 96-well plates (Nunc) unless otherwise stated.

Cell viability was determined using the CellTiter $96^{\circledR}$ AQueous One Solution Cell Proliferation Assay kit (Promega). Briefly, the well plate and its contents were equilibrated to room temperature. Then, $20 \mu \mathrm{L}$ of the CellTiter $96^{\circledR}$ AQueous One reagent was added to each well. The contents were briefly mixed on an orbital shaker and then incubated at $37^{\circ} \mathrm{C}$ in a $5 \% \mathrm{CO}_{2}$ atmosphere for $1-4 \mathrm{~h}$. The absorbance signal was recorded at $490 \mathrm{~nm}$ using a microplate reader (MTP500; Corona Electric, Hitachinaka, Japan). The assay was repeated three times in triplicate. The results are given as the mean \pm standard error of the mean (SEM; $n=3$ ) of three independent experiments.

\section{Invasion and intracellular replication assessment by immunofluorescence (IF) staining \\ Invasion assay}

Invasion assays were performed as described by Kieschnick et $\mathrm{al}^{23}$ and Sugi et al. ${ }^{24}$ Briefly, freshly harvested and purified parasites $\left(1 \times 10^{5}\right)$ were inoculated onto a monolayer of $\mathrm{HFF}$ cells on a coverslip for $1 \mathrm{~h}$ at $37^{\circ} \mathrm{C}$ with the different NP solutions (reconstituted in fresh medium prior to use) or medium only to serve as mock treatment. Following invasion, the cells were washed three times with ice-cold PBS, and extracellular parasites were stained with rabbit anti-T. gondii RH strain polyclonal antibodies $(1: 1,000)$ (Bio-Rad Laboratories, Hercules, CA, USA) in PBS containing $2 \%$ FCS for $30 \mathrm{~min}$ on ice. The cells were then washed three times with PBS containing $2 \%$ FCS, fixed with $4 \%$ paraformaldehyde in PBS, and permeabilized with ice-cold methanol for $20 \mathrm{~min}$.
Then, the cells were washed three times with $2 \%$ FCS-PBS and further stained with monoclonal mouse anti-SAG1 antibodies $(1: 1,000)$ (HyTest, Turku, Finland) in PBS containing $2 \%$ FCS. Secondary staining was achieved by antibody solutions containing Alexa 488 goat antimouse and Alexa 594 goat antirabbit and DAPI for nuclear staining $(1: 1,000)$ in $2 \%$ FCS-PBS. The cells were visualized with a fluorescence microscopy (Eclipse E400; Nikon, Tokyo, Japan). At least 50 microscopic fields were chosen at random; extracellular parasites were detected using the rabbit anti-Toxoplasma antibodies, and the total parasites were detected with the mouse anti-SAG1 antibodies.

\section{Intracellular replication assessment by IF staining}

Intracellular replication assays were performed as described by Sugi et al. ${ }^{24}$ Briefly, purified parasites $\left(1 \times 10^{5}\right)$ were inoculated onto growing HFF monolayers on a coverslip and incubated at $37^{\circ} \mathrm{C}$ for $1 \mathrm{~h}$ to allow invasion. Cells were washed three times with warm culture medium to remove uninvaded parasites and were then incubated for different time periods $(12,16,18$, and $24 \mathrm{~h})$ in medium containing NP solutions or medium only (mock treatment). After each incubation time period, the cells were fixed with $4 \%$ paraformaldehyde in PBS for $30 \mathrm{~min}$ at room temperature, permeabilized with ice-cold methanol for $20 \mathrm{~min}$ at room temperature, and then washed three times with PBS. The cells were blocked with $2 \%$ BSA in $0.1 \%$ Tween $20-\mathrm{PBS}$ for $1 \mathrm{~h}$ at room temperature. The primary antibody, monoclonal mouse anti-SAG1 $(1: 1,000)$ in blocking buffer $(0.1 \%$ Tween $20-\mathrm{PBS})$, was added and incubated for $30 \mathrm{~min}$ at $37^{\circ} \mathrm{C}$. The cells were then washed three times ( 5 min between each wash) with blocking buffer. Secondary antibody solutions containing Alexa 546 goat antimouse and DAPI for nuclear staining in blocking buffer were added and incubated for $30 \mathrm{~min}$ at $37^{\circ} \mathrm{C}$. Coverslips were washed three times with PBS and viewed by fluorescence microscopy (Eclipse E400). At least 50 microscopic fields were chosen at random to count the parasitophorous vacuoles. Average numbers of tachyzoites per vacuole were calculated.

\section{Reversibility}

The reversibility of effect was assessed as described by Kamau et al. ${ }^{4}$ Briefly, invading parasites were grown in the presence of NP solutions (reconstituted in culture medium) for 12 and $24 \mathrm{~h}$. The medium was removed, and the monolayer was washed thoroughly before adding fresh medium. The plate was then incubated in the absence of the NPs for another $24 \mathrm{~h}$, and the growth curve was determined by 
measuring the $\beta$-gal activity using the luminescent reporter assay. In parallel, after each incubation (12 and $24 \mathrm{~h}$ ) of invading parasites in the presence of the NP solutions, parasite viability was determined on the basis of luminescence to compare and evaluate the ability of the parasites to recover from the treatment. Three independent assays were performed.

\section{Infectivity and likely cellular target}

A procedure previously described by Kamau et $\mathrm{al}^{4}$ was used. In brief, freshly lysed and purified parasites were treated for either 24 or $48 \mathrm{~h}$ at $37^{\circ} \mathrm{C}$ in $5 \% \mathrm{CO}_{2}$ with either medium only or an NP solution (reconstituted in culture medium) and then washed three times with culture medium to remove the NPs. The treated parasites were used to infect fresh HFF monolayers by incubating them with the cells for $48 \mathrm{~h}$, prior to assaying for growth using the luminescence reporter assay. Extracellular parasites were incubated with the various NPs for $12 \mathrm{~h}$ after which the parasites were harvested and used for infectivity tests as described earlier.

To determine whether effects on parasite growth were due to effects on the host cell, uninfected HFF monolayers were pretreated for $24 \mathrm{~h}$ at $37^{\circ} \mathrm{C}$ in $5 \% \mathrm{CO}_{2}$ with either medium only or an NP solution. The pretreated host monolayers were washed thoroughly with fresh medium. Freshly lysed and purified parasites were then allowed to invade the pretreated monolayers for $4 \mathrm{~h}$, the monolayer was washed three times with medium, and parasite growth was allowed to proceed for $24 \mathrm{~h}$. Growth curves were determined using the luminescence reporter assay. In parallel, media-pretreated host cell monolayers were infected with parasites in a similar fashion, but the NP solutions were added $4 \mathrm{~h}$ postinvasion and incubated for $24 \mathrm{~h}$. This allowed comparisons of the effects of the NPs after pretreatment to the effects of having the NPs present for the entire incubation period.

\section{Measurement of intracellular ROS}

Measurement of intracellular ROS was based on the intracellular peroxide-dependent oxidation of $2^{\prime}, 7^{\prime}$-dichlorodihydrofluorescein diacetate $\left(\mathrm{H}_{2} \mathrm{DCF}-\mathrm{DA}\right.$; Sigma, St Louis, MO, USA) to form the fluorescent compound $2^{\prime}, 7^{\prime}$-dichlorofluorescein (DCF), as previously described by Warleta et al. ${ }^{25}$ Briefly, growing HFF monolayers were infected with purified parasite suspension and incubated for $24 \mathrm{~h}$ at $37^{\circ} \mathrm{C}$. Subsequently, the parasite-infested HFF cells were treated with the NPs and further incubated for $8 \mathrm{~h}$ at $37^{\circ} \mathrm{C}$. The parasites were then harvested, purified, and resuspended in PBS containing the $\mathrm{H}_{2}$ DCF-DA to a final concentration of
$100 \mu \mathrm{M}$. The parasite suspension containing the fluorescent dye was incubated for $30-60 \mathrm{~min}$ at $37^{\circ} \mathrm{C}$. Fluorescence acquisition was then assessed using a spectrofluorometer (Corona Electric) with excitation set at $485 \mathrm{~nm}$ and emission at $530 \mathrm{~nm} . \mathrm{H}_{2} \mathrm{O}_{2}$ was included as a positive control.

\section{Measurement of the mitochondrial membrane potential}

The measurement of mitochondrial membrane potential was acquired by spectrofluorimetry according to a procedure described by Baracca et al. ${ }^{26}$ Briefly, growing HFF monolayers were infected with purified parasite suspension and incubated for $24 \mathrm{~h}$ at $37^{\circ} \mathrm{C}$. The parasite-infested HFF monolayers were treated with the NPs and further incubated for $8 \mathrm{~h}$ at $37^{\circ} \mathrm{C}$. The parasites were then harvested, purified, and stained with 200 nM MitoRed (Dojindo Molecular Technologies Inc, Kumamoto, Japan) by following the manufacturer's protocol. Fluorescence acquisition was assessed using a spectrofluorometer with excitation set at $560 \mathrm{~nm}$ and emission at $580 \mathrm{~nm}$.

\section{Data analysis}

Data were analyzed using a one-way ANOVA (GraphPad Software Inc, San Diego, CA, USA) and are presented as the mean \pm SEM. Comparisons among groups were determined using a one-way ANOVA and Dunnett's posttest. $P$-value $<0.05$ was considered to be statistically significant. The $\mathrm{EC}_{50}$ was estimated by plotting NP concentrations versus the percentages of $T$. gondii viability, and the $\mathrm{IC}_{50}$ was estimated by plotting NP concentrations versus the percentages of cell viability. All the experiments were developed in the same conditions thrice separately, and the results were expressed as the mean \pm SEM. Selectivity indexes (SIs) were also estimated. The analyses were carried out using Prism 5 (Graphpad Software Inc), whereas nonlinear regression analysis was used to fit the curve. All experiments were performed in triplicate and carried out three times independently unless otherwise stated.

\section{Results}

\section{NPs significantly reduced the parasite growth}

In our preliminary experiments, we sought to establish the antiparasitic activity and host cell cytotoxic potential of the metal NPs. Therefore, first, we screened the NPs (AuNPs, AgNPs, and PtNPs) at various concentrations between 0.01 and $1,000 \mu \mathrm{g} / \mathrm{mL}$ for anti-T. gondii activity by incubating freshly lysed and purified parasites with the NPs in growing monolayers for $48 \mathrm{~h}$. Parasite growth curves were determined 
by measuring the $\beta$-gal activity of the viable parasites using a luciferase reporter assay. The NPs significantly reduced the parasite viability by $>90 \%$ (Figure 1) with $\mathrm{EC}_{50}$ values of $\leq 7, \leq 1$, and $\leq 100 \mu \mathrm{g} / \mathrm{mL}$ for AuNPs, AgNPs, and PtNPs, respectively (Table 1). AuNPs and AgNPs showed a stronger anti- $T$. gondii activity relative to that of PtNPs, consistent with other studies, ${ }^{14-17}$ that have reported antimicrobial and/or antiparasitic properties of AuNPs and AgNPs; similar studies using PtNPs were scarce in the literature. A reference drug (sulfadiazine, used to treat active toxoplasmosis), which was included as a positive control, decreased the parasite viability by $>95 \%$. This is consistent with published data ${ }^{27}$ and thus supports the validity of our screening assay. The estimated $\mathrm{IC}_{50}$ values in our host cell cytotoxicity studies were $\geq 260, \geq 48$, and $\geq 2,000 \mu \mathrm{g} / \mathrm{mL}$ for AuNPs, AgNPs, and PtNPs, respectively. Estimation of the ratio of the host cell cytotoxic $\mathrm{IC}_{50}$ value to the antiparasite $\mathrm{EC}_{50}$ value as a measure of the therapeutic or SI was promising, with the SIs of $\geq 30, \geq 40$, and $\geq 20$ for AuNPs, AgNPs, and PtNPs, respectively. Having established the $\mathrm{EC}_{50}$ values for the NPs, we performed further experiments using the single concentrations of 15,10 , and $1,000 \mu \mathrm{g} / \mathrm{mL}$ for AuNPs, AgNPs, and PtNPs, respectively, unless otherwise stated. Having determined that the NPs at these concentrations showed no host cell toxicity (Figure 2A), we screened them at these concentrations for $T$. gondii growth inhibition using the $\beta$-gal expressing luciferase reporter system. The results showed that the NPs induced $>90 \%$ growth inhibition (Figure 2B). Next, we sought to determine whether the parasite invasion and growth processes were affected by treatment with NPs, using the luciferase reporter assay system. The data revealed that the NPs reduced parasite growth by $>60 \%$ (Figure 2C) and suggest that the NPs may act during the parasite invasion window. This finding could serve as an indirect measure of the parasite invasion efficiency in the presence of NPs. The data from the invasion and growth assay, in which the NPs were present throughout the $48 \mathrm{~h}$ incubation period, showed a $>90 \%$ reduction in parasite viability relative to
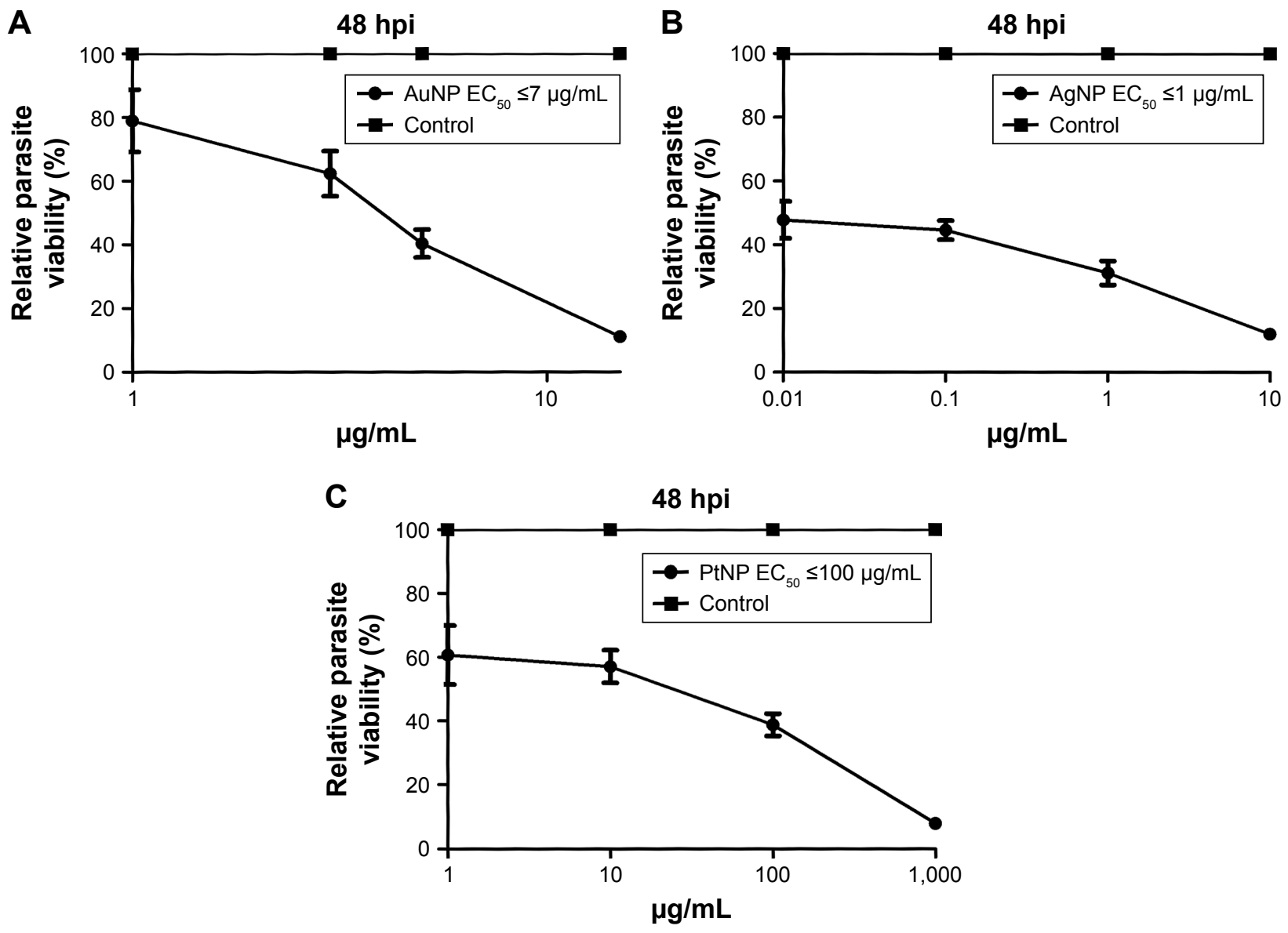

Figure I Preliminary screening of the nanoparticles at various concentrations for anti-Toxoplasma gondii activity to determine $\mathrm{EC}_{50}$ values. Notes: (A) AuNP. (B) AgNP. (C) PtNP. Experiment was in triplicate and performed three times independently.

Abbreviations: AgNP, silver nanoparticle; AuNP, gold nanoparticle; $\mathrm{EC}_{50}$, concentration that inhibits $50 \%$ of Toxoplasma gondii growth; PtNP, platinum nanoparticle; SEM, standard error of the mean. 
Table I Inorganic nanoparticles show strong activity against Toxoplasma gondii versus a host cell

\begin{tabular}{llll}
\hline $\begin{array}{l}\text { Treatment } \\
\text { group }\end{array}$ & $\begin{array}{l}\text { EC }_{50} \text { value } \\
(\mathbf{R H}-2 \mathrm{~F})(\mu \mathrm{g} / \mathrm{mL})\end{array}$ & $\begin{array}{l}\mathrm{IC}_{50}(\mathrm{HFF}) \\
(\mu \mathrm{g} / \mathrm{mL})\end{array}$ & SI \\
\hline AuNP & $\leq 7$ & $\geq 260$ & $\geq 30$ \\
AgNP & $\leq 1$ & $\geq 48$ & $\geq 40$ \\
PtNP & $\leq 100$ & $\geq 2,000$ & $\geq 20$ \\
\hline
\end{tabular}

Notes: Values are expressed as the mean \pm SEM $(n=3)$. Each experiment was in triplicate and performed three times independently. $\mathrm{RH}-2 \mathrm{~F}$ is a strain of Toxoplasma gondii.

Abbreviations: AgNP, silver nanoparticle; AuNP, gold nanoparticle; $\mathrm{EC}_{50}$, concentration that inhibits $50 \%$ of Toxoplasma gondii growth; HFF, human foreskin fibroblast; $I \mathrm{IC}_{50}$, concentration that produces $50 \%$ of cellular inhibition; PtNP, platinum nanoparticle; SEM, standard error of the mean; Sl, selectivity index.

mock treatment (Figure 2D). The results are similar to those obtained for the growth only assay, in which the purified parasites were allowed to invade the host monolayers for $1 \mathrm{~h}$ before the addition of the NPs. To further confirm that the
NPs have a direct anti-T. gondii activity, we incubated freshly lysed and purified parasites with the NPs for $12 \mathrm{~h}$ extracellularly. Relative to the mock treatment, the NPs significantly $(P<0.05)$ reduced the parasite viability (Figure 2E), comparable to the results obtained when the parasite viability screening was done using the host monolayer, lending credence to the antiparasitic action of the NPs. Efforts to determine whether the NPs were inducing cyst formation rather than killing the parasite (using CST1 staining; data not shown) revealed the absence of cyst formation and thus supported the concept that treatment with NPs killed the parasites.

\section{Poor infectivity and recovery following NP treatment}

First, we sought to assess whether the NP-treated parasites could sustain their ability to infect fresh host monolayers.
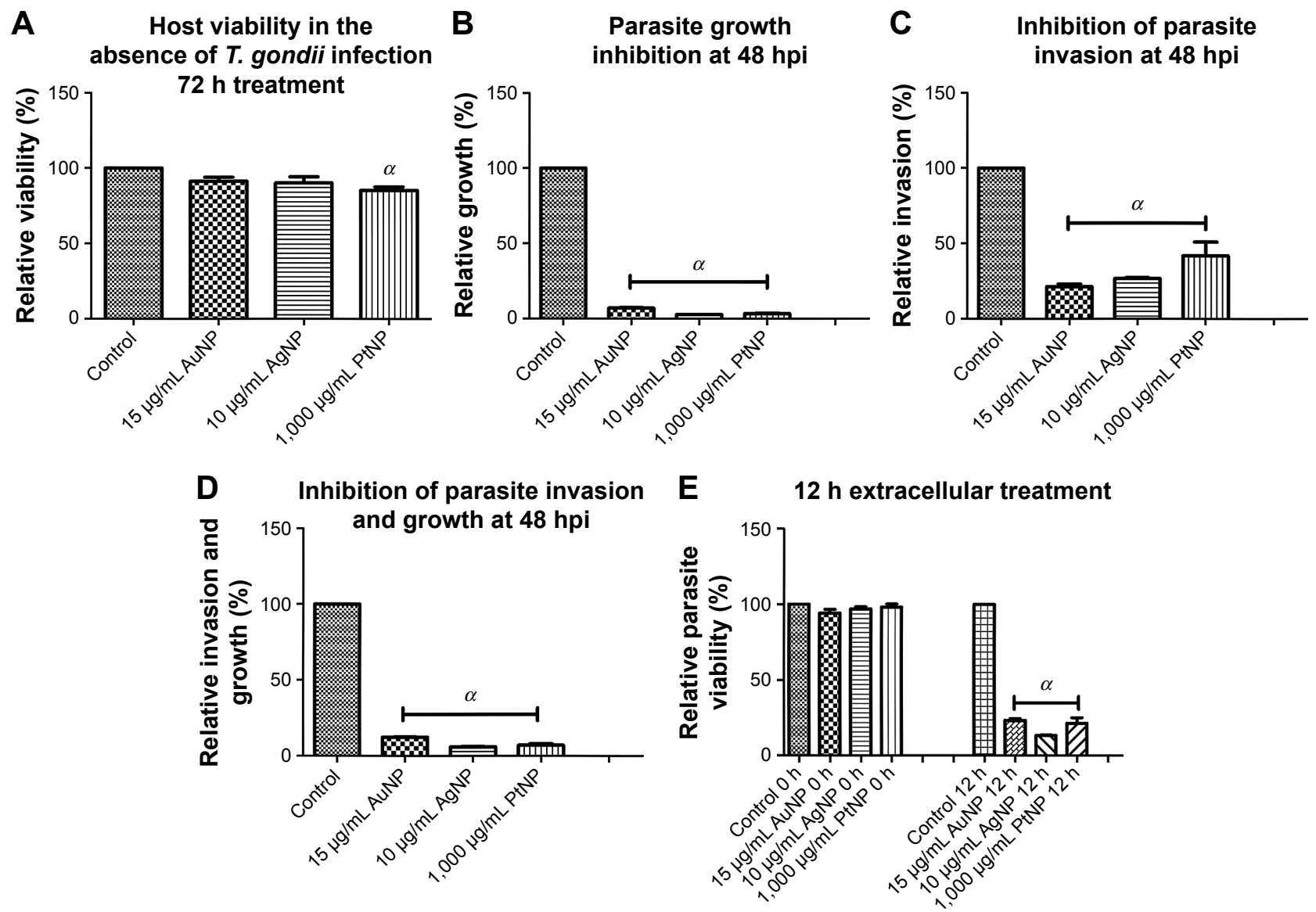

Figure 2 (A) In the absence of Toxoplasma gondii infection, the host monolayers were treated with NPs at the effective anti-T. gondii concentration and cell viability was determined after $72 \mathrm{~h}$ incubation. (B) Freshly lysed and purified parasites were allowed to invade growing HFF cells for I h. The infection medium was then removed, and fresh medium containing NPs was added. After $48 \mathrm{~h}$ incubation, growth inhibition was determined using a luciferase reporter assay. (C) Purified parasite suspension and NPs were added to growing HFFs and incubated for I $\mathrm{h}$. Then the infection medium was replaced with fresh medium, and the cells were incubated for an additional $48 \mathrm{~h}$. Growth inhibition was determined using a luciferase reporter assay. (D) Purified parasite suspension and NPs were added to growing HFF monolayers and incubated for $48 \mathrm{~h}$ before growth curves were prepared based on data from a luciferase reporter assay system. (E) Freshly purified parasites were incubated with NPs under extracellular conditions for $12 \mathrm{~h}$. Notes: The data are expressed as the mean \pm SEM $(n=3)$. $\alpha$ is significant at $P<0.05$ relative to the control. Experiment was in triplicate and performed three times independently.

Abbreviations: AgNP, silver nanoparticle; AuNP, gold nanoparticle; HFFs, human foreskin fibroblasts; PtNP, platinum nanoparticle; SEM, standard error of the mean; NPs, nanoparticles. 
Freshly lysed and purified parasites were incubated with the NPs in growing host monolayers for 24 and $48 \mathrm{~h}$. At each time point, the parasites were harvested and used to infect fresh host cells. After $48 \mathrm{~h}$ incubation, the parasite viability was determined. The results showed that treatment with the NPs at both time points negatively $(P<0.05)$ impacted parasite infectivity (Figure 3A and B). NP treatment produced a $>75 \%$ reduction in parasite infectivity potential relative to mock treatment. In parallel, for comparison, we determined the parasite infectivity potential using extracellular parasites exposed to the NPs for $12 \mathrm{~h}$. The data showed a similar trend of significant depression of the parasite infectivity by the NPs (Figure 3C). This finding thus confirms the antiparasitic action of the NPs. The poor infectivity potential exhibited by the parasites after treatment with the NPs could indicate unlikely reversible antiparasitic actions. Therefore, we evaluated whether the antiparasitic effect of NPs was reversible. Freshly lysed and purified parasites were incubated with the NPs in growing host monolayers for 12 and $24 \mathrm{~h}$. At each time point, the NPs were removed and replaced with fresh medium, and the plates were incubated for a further $24 \mathrm{~h}$ after which parasite viability was determined. In parallel, parasite viability at 12 and $24 \mathrm{~h}$ of NP or mock treatment was determined to serve as the baseline for comparison. The data revealed a marginal recovery of $<10 \%$ following $24 \mathrm{~h}$ of NP treatment. Conversely, a slightly higher recovery of $<25 \%$ was observed for the $12 \mathrm{~h}$ NP treatment (Figure 4A and B). These data suggest that the NPs exhibit a minimally reversible but time-dependent antiparasitic action. It thus appears that the incubation time of the NPs with the parasites may play a role in determining whether the antiparasitic action may be reversible. Overall, our results indicate that the poor parasite infectivity potential may preclude the reversibility of the antiparasitic effects of the NPs.

\section{The antiparasitic action of the NPs may be due, in part, to a parasite target}

We then sought to determine whether the NPs affected parasite viability through a host or parasite target. Growing
A

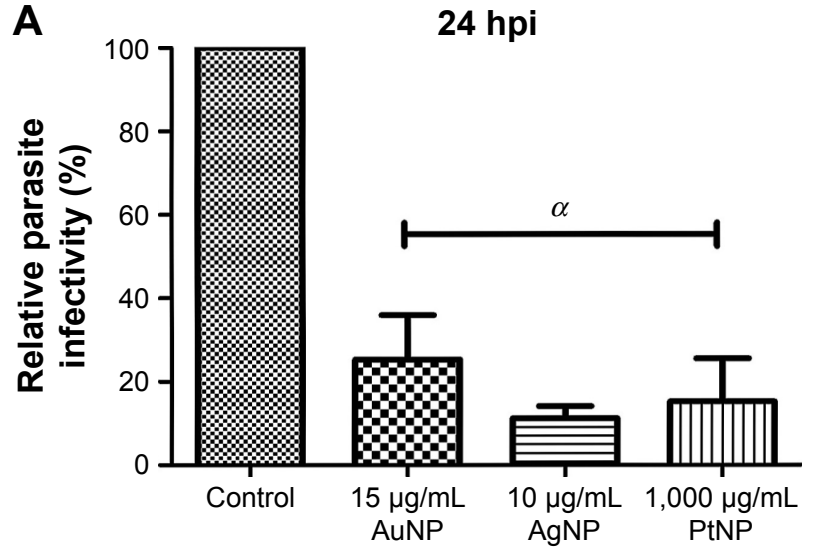

B

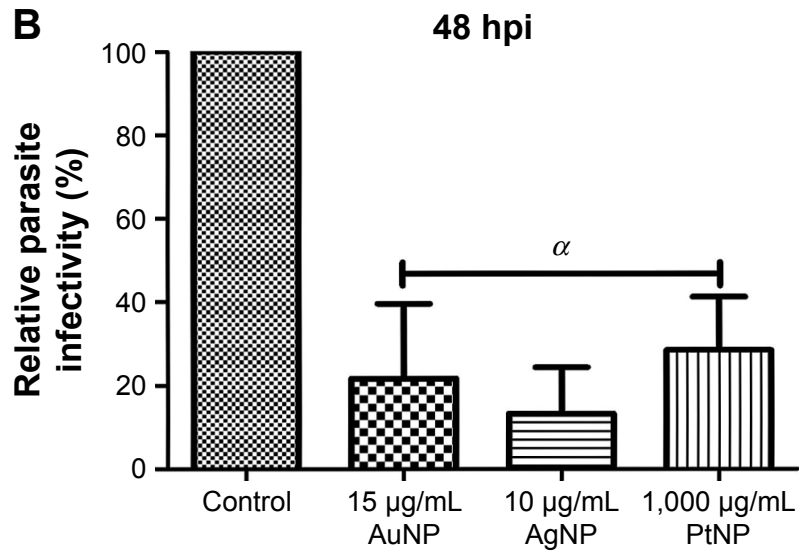

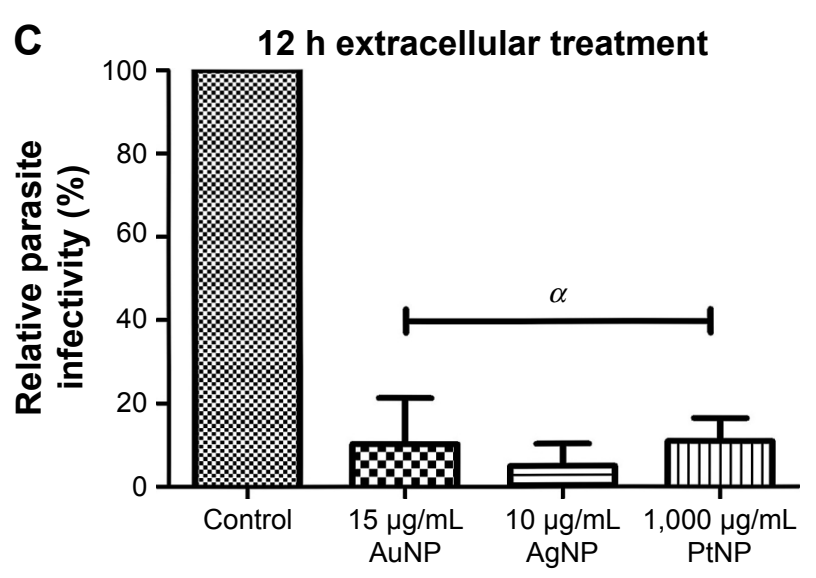

Figure 3 (A) Invading parasites were treated with nanoparticles (NPs) for $24 \mathrm{~h}$ after which the parasites were harvested and used to infect fresh HFF monolayers. (B) Invading parasites were treated with NPs for $48 \mathrm{~h}$ after which the parasites were harvested and used to infect fresh HFF monolayers. (C) Extracellular parasites were treated with NPs for $12 \mathrm{~h}$ after which the parasites were harvested and used to infect fresh HFF monolayers.

Notes: Parasite infectivity was determined after $48 \mathrm{~h}$ incubation. The data are expressed as the mean $\pm \mathrm{SEM}$ ( $\mathrm{n}=3$ ). $\alpha$ is significant at $\mathrm{P}<0.05$ relative to the control. Experiment was in triplicate and performed three times independently.

Abbreviations: AgNP, silver nanoparticle; AuNP, gold nanoparticle; HFF, human foreskin fibroblast; PtNP, platinum nanoparticle; SEM, standard error of the mean. 

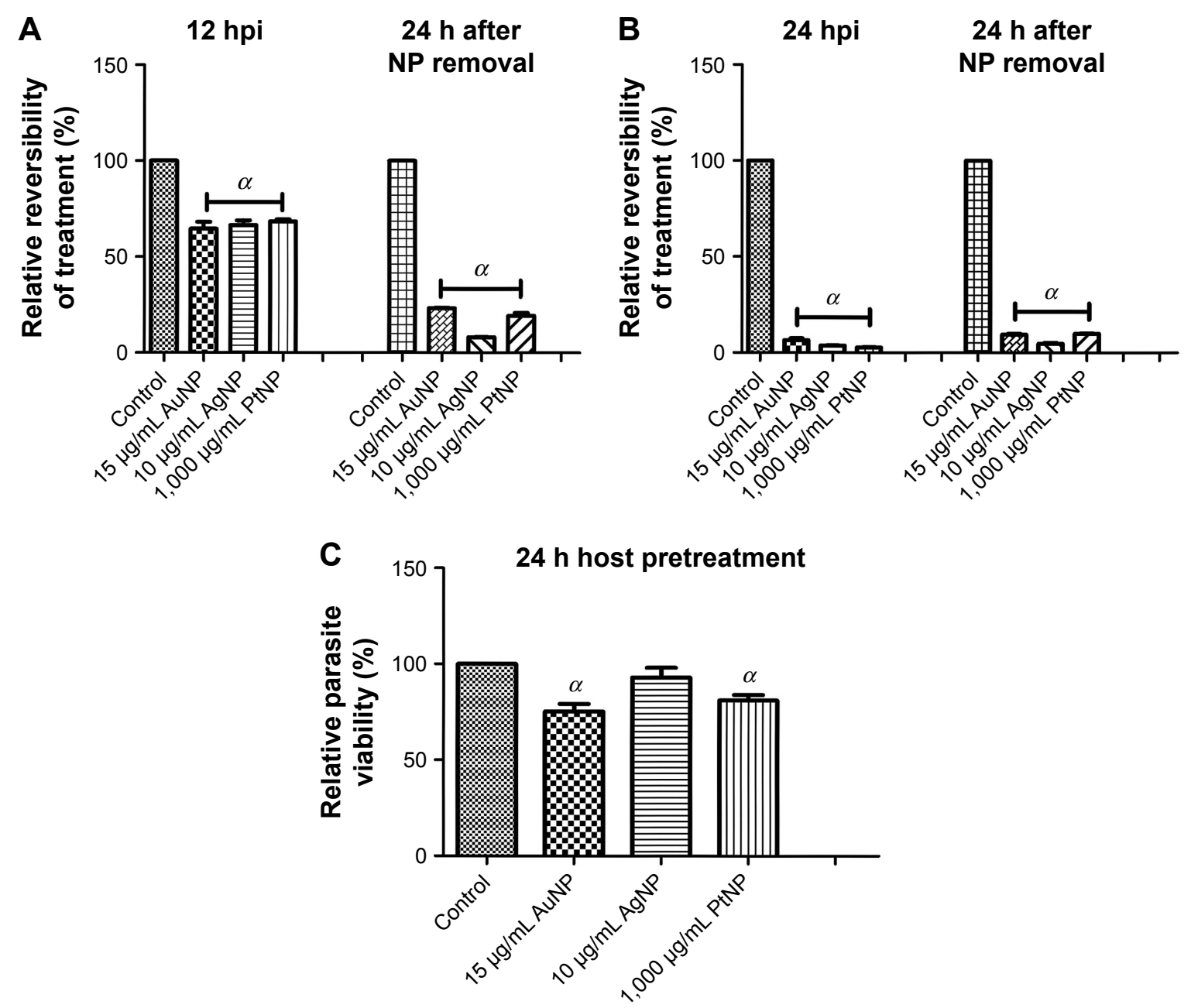

Figure 4 (A) Invading parasites were treated with NPs for $12 \mathrm{~h}$ after which the medium was removed, the cells were washed thoroughly, and the medium was replaced. The cells were incubated for another $24 \mathrm{~h}$ after which the reversibility of treatment was evaluated through growth curve determinations. (B) Invading parasites were treated with NPs for $24 \mathrm{~h}$ after which the medium was removed, the cells were washed thoroughly, and the medium was replaced. The cells were incubated for another $24 \mathrm{~h}$ after which the reversibility of treatment was evaluated through growth curve determinations. (C) Growing HFF monolayers were pretreated with NPs for $24 \mathrm{~h}$ after which the pretreated host cells were washed thoroughly with fresh medium. Freshly purified parasites were then allowed to invade and infect the pretreated HFF monolayers, and the parasite growth potential was determined after $24 \mathrm{~h}$.

Notes: The data are expressed as the mean \pm SEM ( $n=3)$. $\alpha$ is significant at $P<0.05$ relative to the control. Experiment was in triplicate and performed three times independently.

Abbreviations: AgNP, silver nanoparticle; AuNP, gold nanoparticle; HFF, human foreskin fibroblast; NP, nanoparticle; PtNP, platinum nanoparticle; SEM, standard error of the mean.

host cell monolayers were pretreated with the NPs for $24 \mathrm{~h}$, and then freshly lysed and purified parasites were allowed to infect the pretreated host cells. After $24 \mathrm{~h}$ incubation, the parasite viability was determined. The data revealed $>75 \%$ parasite growth in the host cells pretreated with the NPs relative to the mock pretreatment (Figure 4C). Although these findings suggest that the antiparasitic effects of NPs may be linked to a parasite target, we could not rule out the possibility that a host cell target also contributed to the observed anti- $T$. gondii activity of NPs. Of note, $T$. gondii growth in the host monolayers pretreated with AuNPs and PtNPs was significantly reduced by $\leq 20 \%$.

\section{NPs affect parasite invasion and replication}

Toward unraveling the mode of the antiparasitic action of NPs, we sought to determine whether any stage of the parasite lytic cycle was affected by the NPs. The parasite lytic cycle involves the following three repeated stages: invasion, replication, and egress. We assessed the effect of NPs on invasion and intracellular replication. The IF staining was used to assess the ability of the parasites to invade growing host monolayers and to determine the parasite number per vacuole in the presence of the NPs. The results revealed a significant reduction in parasite ability to invade growing 
host monolayers by $>65,>60$, and $>50 \%$ for AuNPs, AgNPs, and PtNPs, respectively (Figure 5A). Similarly, the NPs caused $>67 \%$ depletion in the average replication of parasites per vacuole (at least 100 vacuoles were counted per experiment) relative to the mock treatment (Figure 5B). These findings corroborate the data obtained from the invasion and growth assays using the luciferase reporter systems in which the NPs caused a $>90 \%$ parasite growth inhibition. Further analyses at different time points $(12,16,18$, and $24 \mathrm{~h})$ showed that NP treatment resulted in a time-dependent reduction in the number of parasites per vacuole (Figure 6A-D). Moreover, the highest number of parasites per vacuole was $<8$ for the NPs relative to the mock treatment, for which the parasite number steadily increased with incubation time.

In support of the time-dependent depression of the capacity of the parasites to replicate, at the $24 \mathrm{~h}$ time point, the treatment with the NPs led to $<4$ parasites per vacuole (Figure 7A-C), which was in stark contrast to the mock treatment, where the number of parasites per vacuole gradually advanced to $>24$.

\section{Anti-T. gondii activity of NPs may be linked to altered redox status via intracellular production of ROS}

Having established that the NPs possess anti-T. gondii activity, we sought to understand their likely mode of the antiparasitic action. First, we tested whether ROS play a role in the parasite killing capability of the NPs. In this experiment, we added an antioxidant, Trolox, to the NP antiparasite screening assay. The data showed that the antiparasitic effect of the NPs was mitigated in the presence of Trolox (Figure 8A-C). Although at the highest concentration $(1,000 \mu \mathrm{g} / \mathrm{mL})$, the addition of Trolox had no visible effect on the antiparasitic action of PtNPs. Nonetheless, the results indicate that the $\mathrm{EC}_{50}$ values for the NPs were increased in the presence of Trolox compared to that in the absence of Trolox. These findings implicate oxidative stress in the anti- $T$. gondii effect of the NPs. Although the reversal of the antiparasitic action of NPs through the addition of Trolox indicated a connection to oxidative stress, it was not a direct evaluation of the production of ROS. Therefore, we sought to directly determine whether ROS was produced during NP treatment of $T$. gondii. We monitored the production of ROS using $\mathrm{H}_{2}$ DCF-DA, a cell-permeable reduced fluorescein that is nonfluorescent until its acetate groups are removed by intracellular esterases during oxidation. Our results showed that AgNPs and PtNPs caused $>100 \%$ ROS production relative to the nontreated control (Figure $9 \mathrm{~A}$ and B). Similar results ( $>200 \%$ over the untreated control) were obtained following $\mathrm{H}_{2} \mathrm{O}_{2}$ treatment (data not shown), validating the ROS detection assay. Conversely, the level of ROS production induced by AuNP treatment was higher than the untreated control but not statistically significant. We then asked whether the NP-induced ROS production was sustained after removing the NPs. The results showed that ROS production was sustained following treatment with AuNPs or AgNPs for $12 \mathrm{~h}$ after removing the NPs, suggesting that these NPs had a negative impact on the parasites.

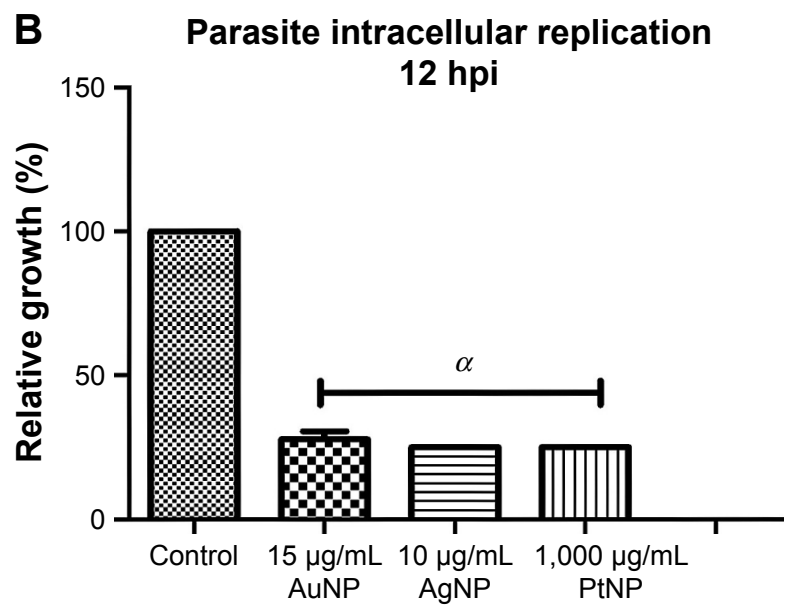

Figure 5 IF staining of infected HFF cells that were treated with nanoparticles (NPs).

Notes: (A) Freshly purified parasite suspension and NPs were added to growing HFF monolayers on coverslips. Invasion was allowed for I h before IF staining. (B) Freshly purified parasite suspensions were added to growing HFF monolayers on coverslips. Invasion was allowed for I h after which the infection medium was replaced with fresh medium containing NPs. IF staining of intracellular parasites was carried out after $12 \mathrm{~h}$ incubation. The data are expressed as the mean $\pm \mathrm{SEM}$ ( $\mathrm{n}=3$ ). $\alpha$ is significant at $P<0.05$ relative to the control. Experiment was performed three times independently.

Abbreviations: AgNP, silver nanoparticle; AuNP, gold nanoparticle; HFF, human foreskin fibroblast; IF, immunofluorescence; PtNP, platinum nanoparticle; SEM, standard error of the mean. 

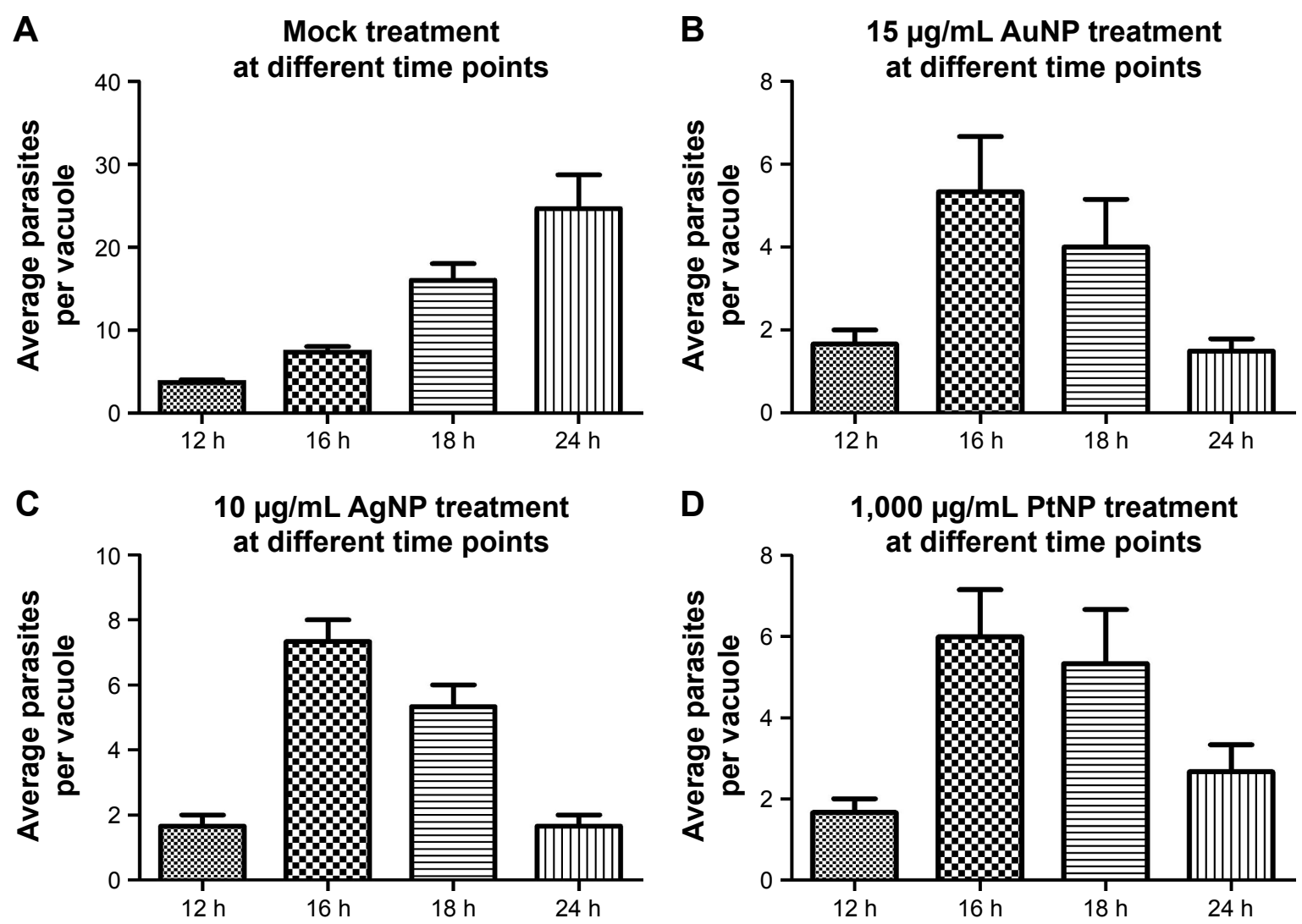

Figure 6 IF staining to determine the number of parasites per vacuole following treatment with nanoparticles at the effective anti-Toxoplasma gondii concentration for different time periods.

Notes: (A) Mock treatment. (B) AuNP treatment. (C) AgNP treatment. (D) PtNP treatment. The data are expressed as the mean \pm SEM ( $n=3$ ). Experiment was performed three times independently.

Abbreviations: AgNP, silver nanoparticle; AuNP, gold nanoparticle; IF, immunofluorescence; PtNP, platinum nanoparticle; SEM, standard error of the mean.

\section{NPs decrease the parasite mitochondrial membrane potential}

The mitochondria represent a major source of ROS in the cell. Indeed, mitochondria are considered as the major target of excess cellular ROS. We therefore asked whether NPinduced ROS production affected the parasite mitochondria. We used MitoRed to evaluate the parasite mitochondrial membrane potential. MitoRed is a rhodamine-based dye that permeates cell membranes. It localizes in mitochondria and emits fluorescence. The interaction of MitoRed with the mitochondria is dependent on the membrane potential of the mitochondria. The $8 \mathrm{~h}$ treatment with the NPs led to reduced fluorescence intensity (Figure 10A and B), suggesting low accumulation of the rhodamine-based dye in the parasite mitochondria. This finding may indicate that the treatment with the NPs affected the parasite mitochondria by decreasing their membrane potential. It was also found that $12 \mathrm{~h}$ after the NPs had been removed, the parasites may not have recovered from the NP-induced reduction in their mitochondrial membrane potential. This finding further supports the antiparasitic impact of NP treatment on parasite mitochondria.

\section{Discussion}

Our study demonstrates that AuNPs, AgNPs, and PtNPs have a promising anti- $T$. gondii activity without detectable host cell toxicity. We obtained antiparasite $\mathrm{EC}_{50}$ values of $<7,<1$, and $<100 \mu \mathrm{g} / \mathrm{mL}$ for AuNPs, AgNPs, and PtNPs, respectively. Although the published reports of the antimicrobial and/or antiparasitic properties of PtNPs are limited, the $\mathrm{EC}_{50}$ values obtained in our study are well within the range of those reported elsewhere ${ }^{16,17,28-30}$ for the antimicrobial and/or antiparasitic action exhibited by AuNPs and AgNPs. Interestingly, a few of the studies ${ }^{17,29,30}$ reporting the antiparasitic activities of NPs revealed that under the same conditions, AgNPs had a lower $\mathrm{EC}_{50}$ value relative to that of AuNPs. A similar trend was observed in our studies that supports and further strengthens the promising antiparasitic potential of AuNPs and AgNPs. However, for PtNPs, a higher concentration was required to demonstrate the commensurate effective 


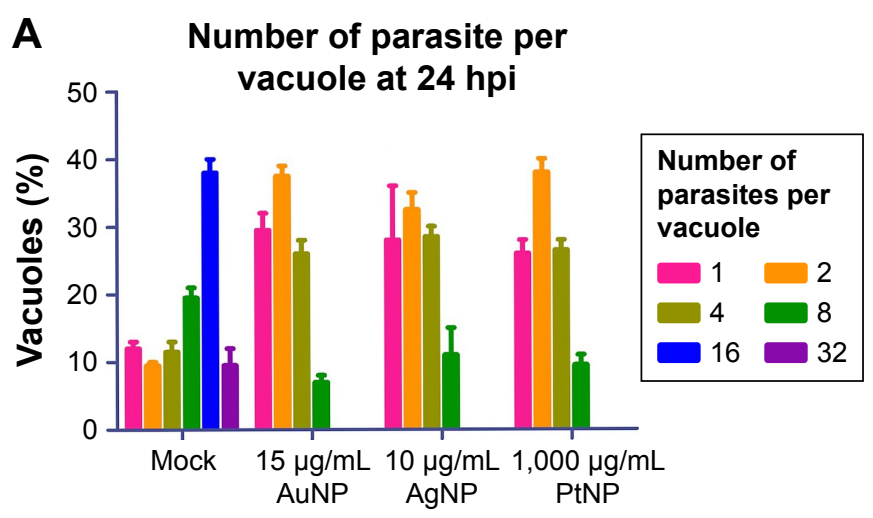

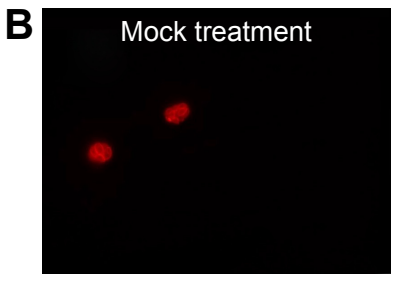

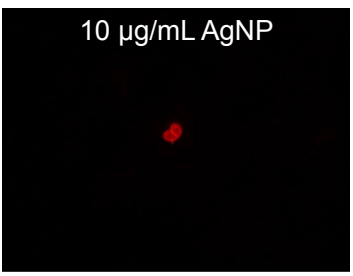

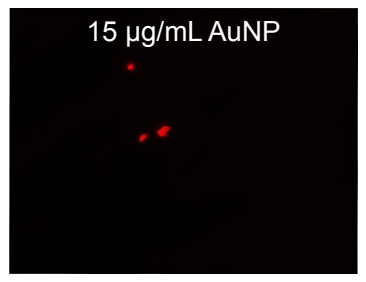

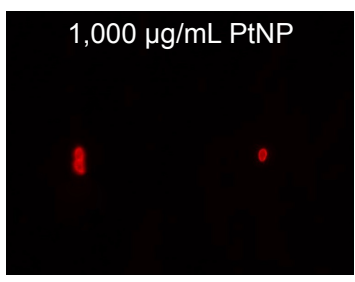

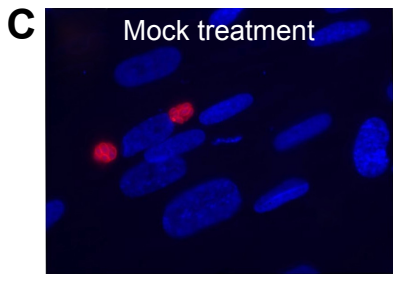

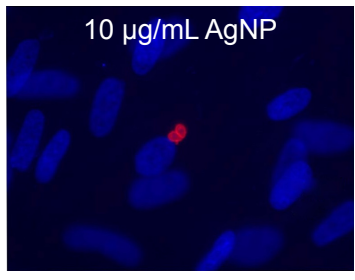

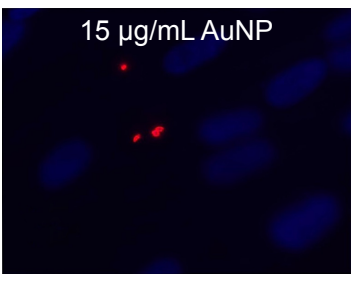

$1,000 \mu \mathrm{g} / \mathrm{mL}$ PtNP

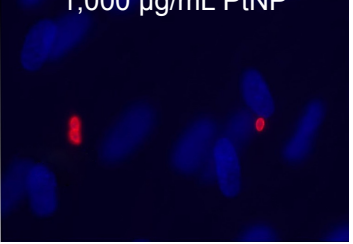

Figure 7 (A) Number of parasites per vacuole at 24 hpi. IF staining to estimate the number of parasites based on 100 parasitophorus vacuoles after treatment with nanoparticles (NPs) at the effective anti-Toxoplasma gondii concentration. The data are expressed as the mean $\pm \mathrm{SEM}$ ( $\mathrm{n}=3$ ). $\alpha$ is significant at $\mathrm{P}<0.05$ relative to the control. Experiment was performed three times independently. (B) Representative fluorescent images showing replicating tachyzoites (RH-2F strain) only. (C) Replicating tachyzoites (RH-2F strain) with nuclear staining (DAPI) for the intracellular replication by IF assay. IF staining was performed at different time points following treatment with nanoparticles at the effective anti-T. gondii concentration. Mock treatment, culture medium only.

Abbreviations: AgNP, silver nanoparticle; AuNP, gold nanoparticle; IF, immunofluorescence; PtNP, platinum nanoparticle; SEM, standard error of the mean.

anti-T. gondii activity. The absence of appreciable host cell toxicity induced by the NPs at the effective anti- $T$. gondii concentrations supports further exploration of the selective antiparasitic action of NPs. The estimated therapeutic or SI revealed $\geq 13$-fold more activity against the parasite versus the host cell for AuNPs and AgNPs, whereas it was $\geq 7$ for PtNPs. This fact coupled with the absence of host cell toxicity induced by the NPs at the effective anti- $T$. gondii concentrations as well as the $\geq 75 \%$ parasite growth in host monolayers pretreated with the NPs suggests that these NPs have a partially selective antiparasitic action. Moreover, findings obtained when extracellular tachyzoites were incubated for $12 \mathrm{~h}$ with the NPs not only reinforce that the NPs have anti-T. gondii activity but also confirm previous reports ${ }^{16,17}$ demonstrating the antiparasitic potential of NPs. In addition, the poor recovery of parasites as well as the marginal parasite infectivity after treatment with NPs supports the further exploration of NPs as anti-infective agents. Together, these data show that NPs have favorable anti-T. gondii potential and thus support several earlier reports that demonstrated the antiCryptosporidium parvum, ${ }^{28}$ antileishmanial, ${ }^{16}$ antifilarial, ${ }^{30}$ antitrypanosomal, and antimalarial activities ${ }^{17}$ of AgNPs as well as the antiparasitic activity of AuNPs. ${ }^{17,30}$ However, this is the first report to show that AuNPs, AgNPs, and PtNPs can cause the death of $T$. gondii both within the host cell and extracellularly. Our findings attest to the promising potency of the NPs as antimicrobial, ${ }^{14,15}$ antiparasitic, ${ }^{17,29,30}$ and bioactive agents, ${ }^{8,9,18,31}$ and may herald a turning point or revolution in pharmacological therapy for parasitic infections.

Our data confirm that NPs significantly impact the invasion and replication stages of the parasite lytic cycle. These findings are preliminary and may represent early insights into how NPs restrict parasite growth. Although we do not yet know how or which of the processes that lead to parasite invasion of the host cell were impeded by the NPs (eg, association with the host plasma membrane, attachment to the host membrane, and host cell penetration ${ }^{32}$ ), our data provide evidence of a time-dependent reduction in parasite 

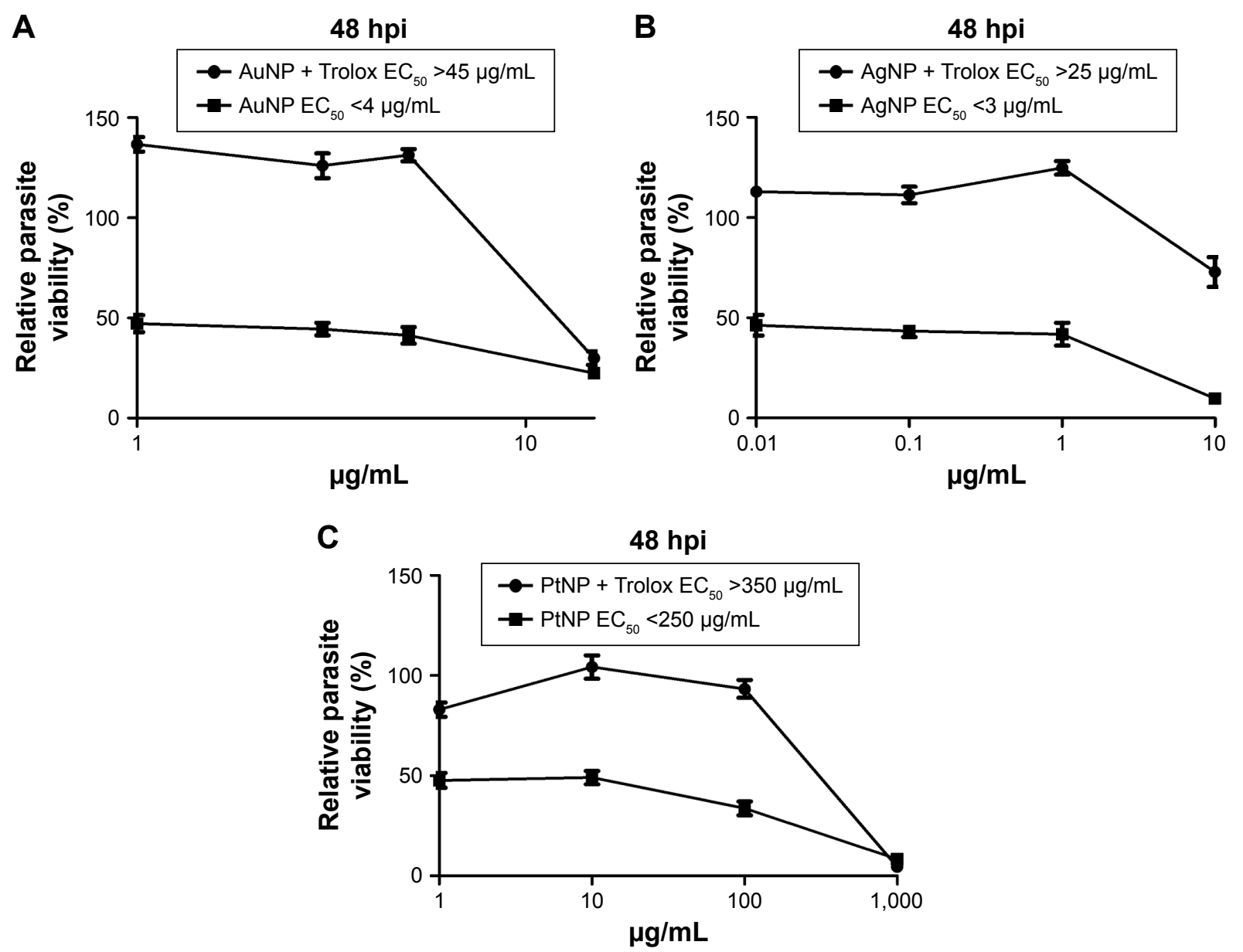

Figure 8 Effect of Trolox on the anti-Toxoplasma gondii activity of the nanoparticles.

Notes: (A) AuNP. (B) AgNP. (C) PtNP. The data are expressed as the mean \pm SEM ( $n=3)$. Experiment was in triplicate and performed three times independently.

Abbreviations: AgNP, silver nanoparticle; AuNP, gold nanoparticle; PtNP, platinum nanoparticle; SEM, standard error of the mean; $\mathrm{C}_{50}$, concentration that inhibits $50 \%$ of Toxoplasma gondii growth.

A

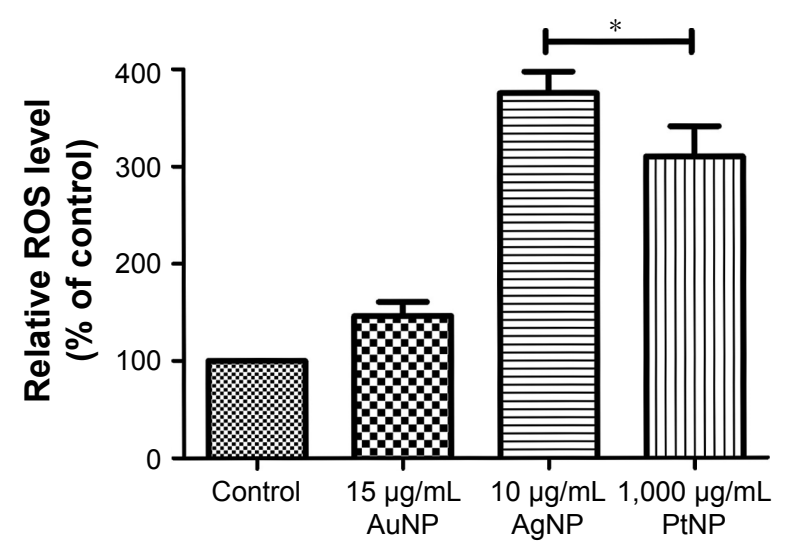

B ROS level $12 \mathrm{~h}$ after NP removal

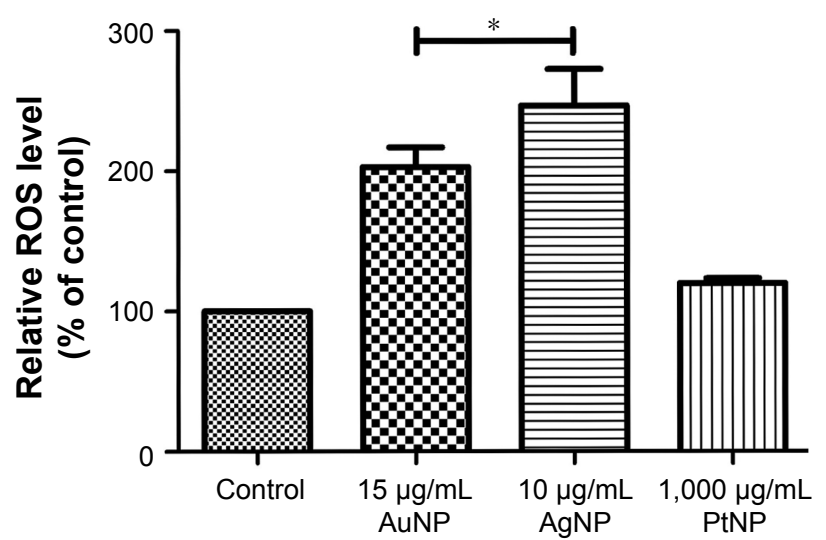

Figure 9 Effect of NPs on parasite redox status by measuring intracellular ROS production.

Notes: (A) The relative ROS level in the parasite was determined after $8 \mathrm{~h}$ of treatment with NPs. (B) The parasite-infected HFF monolayers were treated with NPs for $8 \mathrm{~h}$, after which the treatment was removed. The relative ROS level was determined $12 \mathrm{~h}$ after the removal of the NPs. The data are expressed as the mean \pm SEM ( $=6$ ). The * is significant at $P<0.000$ I relative to the control. Experiment was in triplicate and performed three times independently.

Abbreviations: AgNP, silver nanoparticle; AuNP, gold nanoparticle; NP, nanoparticle; PtNP, platinum nanoparticle; ROS, reactive oxygen species; SEM, standard error of the mean. 
A

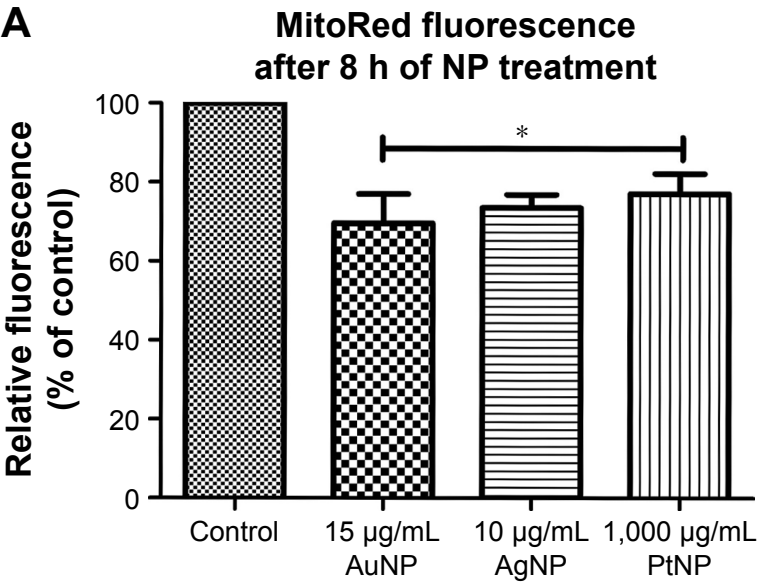

B

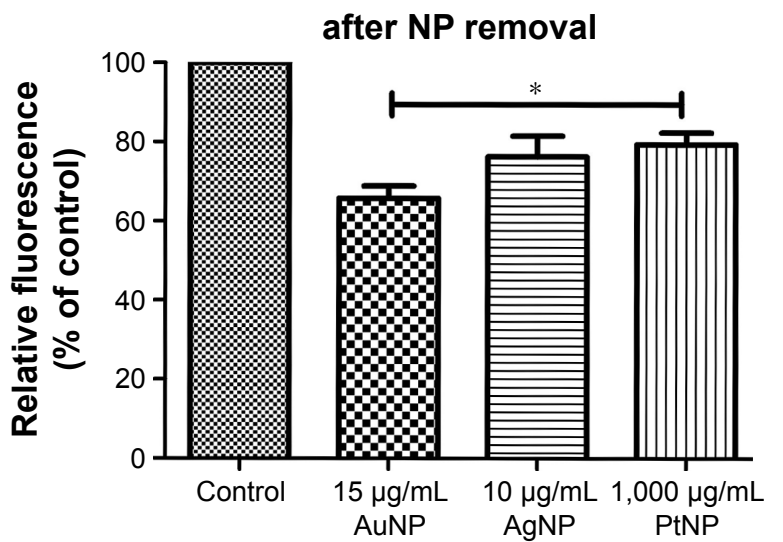

Figure 10 Effect of NPs on parasite mitochondrial membrane potential.

Notes: A cell-permeable rhodamine-based dye (MitoRed), which localizes to mitochondria and emits fluorescence, was used. The interaction of MitoRed with the mitochondria is dependent on the mitochondrial membrane potential. (A) The relative fluorescence intensity of MitoRed in parasite mitochondria after $8 \mathrm{~h}$ of treatment with NPs. (B) The parasite-infected HFF monolayers were treated with NPs for $8 \mathrm{~h}$, after which the treatment was removed. The relative fluorescence intensity of MitoRed in the parasite mitochondria was evaluated $12 \mathrm{~h}$ after removal of the NPs. The data are expressed as the mean \pm SEM ( $=6$ ). The $*$ is significant at $P<0.000 \mathrm{I}$ relative to the control. Experiment was in triplicate and performed three times independently.

Abbreviations: AgNP, silver nanoparticle; AuNP, gold nanoparticle; NP, nanoparticle; PtNP, platinum nanoparticle; SEM, standard error of the mean.

vacuoles and in the number of parasites per vacuole, which, if considered together, suggest that treatment with NPs arrests T. gondii replication rather than slowing it. We are of the opinion that the depleting numbers of parasite vacuoles were unlikely to be a result of premature egress by parasites from the host cell because the host cell numbers did not appear to diminish and extracellular parasites were not observed. These findings suggest that the NPs may have induced a host cellmediated clearance of intracellular parasites through as yet unknown mechanisms.

Our findings also showed a significant reduction in the capacity of the parasites to successfully infect a fresh host after 24 or $48 \mathrm{~h}$ treatment with NPs, even though our data suggest that the antiparasitic effects of NPs may be marginally reversible. The NP-induced paralysis of the potential of parasites to successfully infect fresh host monolayers is not unexpected because it is compatible with previously documented findings of the anti-infective potential of NPs..$^{10,11}$ It is possible that the poor parasite recovery following NP treatment stems from an NP-induced impact on parasite capacity for successful invasion and to a larger extent parasite infectivity. Our data imply that the anti- $T$. gondii action of NPs may be partially linked to their ability to affect the invasion and replication stages of the parasite lytic cycle. Taken together, the antiparasitic actions displayed by the NPs in this study are consistent with published findings on the antimicrobial actions of the NPs reviewed by Yah and Simate $^{33}$ and support the concept of exploring NPs as promising alternative anti- $T$. gondii agents.
Because nanomedicine is still in its infancy, the antimicrobial and/or antiparasitic mode of action of the NPs is largely unknown. Our findings presented here implicate intracellular ROS in the parasite killing action of the NPs. The reversal of the antiparasitic action of NPs by Trolox suggested the involvement of oxidative stress in NP-induced killing of T. gondii; however, ROS measurement through the use of a fluorescent probe provided direct evidence that NP treatment promoted ROS production with a consequent contribution to NP-induced parasite death. These findings are consistent with the few reports that have shown that ROS generation by NPs, including AgNPs and AuNPs, is damaging, leading to the death of Leishmania major ${ }^{29}$ and causing the apoptotic killing of Filaria parasites. ${ }^{30}$

The lack of significant ROS production by AuNPs relative to the control may indicate that AuNP-induced ROS production was not a sudden burst of ROS production but rather a more extended process than that induced by AgNP or PtNP treatment. This concept is supported by the fact that after the NPs had been removed for $12 \mathrm{~h}$, ROS production due to AuNP treatment steadily increased. This finding demonstrates a connection between oxidative affront and the parasite killing potential of AuNPs. Furthermore, the finding that the ROS production induced by AuNPs and AgNPs was sustained for $12 \mathrm{~h}$ after removing the NPs indicates that the oxidative stress caused by the NP treatment may have resulted from extended generation of ROS rather than an immediate burst of ROS. The sustained oxidative stress may have been responsible for the growth arrest as well as for the death 
of the parasite. The sustained oxidative onslaught would be very injurious, causing appreciable damage to cellular biomolecules, and may fuel the antiparasitic potency of the NPs. In contrast, the ROS production after the removal of PtNPs for $12 \mathrm{~h}$ showed a drastic decline. At the moment, we may not be able to explain the reason for this observation. Nonetheless, the protection afforded by the addition of Trolox strongly accentuated the role of oxidative insult in the antiparasitic action of the NPs. Furthermore, the NP-induced ROS production after the removal of the NPs adds evidence in support of the antiparasitic effects of these NPs. Taken together, our findings indicate that the antiparasitic action of the NPs could, in part, be linked to the altered redox status possibly via ROS production. This would not be unexpected given existing reports demonstrating the in vitro and in vivo ROS-generating potential of inorganic NPs, including AuNPs and AgNPs. ${ }^{12,29,30}$ Our data are in full accord with the ROSinducing anti-infective character of NPs. ${ }^{10,11}$

In addition, our finding that treatment with NPs depleted the parasite mitochondrial membrane potential further reinforces our evidence for intracellular ROS production and its culpability in the antiparasitic action. The NP-induced decrease in the parasite mitochondrial membrane potential was sustained, even up to $12 \mathrm{~h}$ after the NPs had been removed. These data are consistent with the recent findings of Charvat and Arrizabalaga, ${ }^{34}$ which indicated that oxidative stress as a consequence of excess cellular ROS production was devastating to the parasite mitochondrial membrane potential and led to the death of $T$. gondii. Furthermore, these findings are consistent with several other reports that have shown that mitochondria are negatively affected by excess cellular ROS production, whereby ROS induces cell death by either promoting intrinsic apoptotic processes and/or activating cellular autophagy, ${ }^{35-37}$ all of which are possible routes to cell death as a result of NP-induced ROS production and/or altered redox status.

The finding that $T$. gondii succumbed to an NP-induced oxidative onslaught provides further evidence to strengthen the support for exploring compounds that can induce oxidative affront and/or interfere with parasite redox biology as a viable approach for the development of antiparasitic agents.

\section{Conclusion}

Our findings identify AuNPs, AgNPs, and PtNPs as promising anti- $T$. gondii candidates. To our knowledge, this is the first evidence of anti-T. gondii activity by AuNPs, AgNPs, and PtNPs. Furthermore, the antiparasitic action of these NPs could be linked, in part, to altered redox status via ROS production. These novel findings add to the growing body of data supporting the need to explore the potential of NPs within the nanomedical community. Further investigations, including in vivo evaluation as well as assessments of the anti-T. gondii potential of surface-modified NPs, are ongoing with a view toward developing a new and effective treatment strategy for acute and/or latent toxoplasmosis.

\section{Acknowledgments}

The study was funded through a Japan Society for the Promotion of Science (JSPS) Fellowship to Dr OS Adeyemi. This study was supported by grants-in-aid for Young Scientists, Exploratory Research, and Scientific Research on Innovative Areas (3308 and 3407) from the Ministry of Education, Culture, Science, Sports, and Technology (MEXT) of Japan; by the Program to Disseminate Tenure Tracking System and the Adaptable \& Seamless Technology Transfer Program through Target-driven R\&D (A-STEP) from the Japan Science and Technology Agency (JST); by the Ito Foundation; and by the Promotion for Young Research Talent and Network from Northern Advancement Center for Science \& Technology (NOASTEC). The authors appreciate Dr Daisuke Kondoh of the Obihiro University of Agriculture and Veterinary Medicine, Japan, for providing assistance with electron microscopy.

\section{Disclosure}

The authors report no conflicts of interest in this work.

\section{References}

1. Beck HP, Blake D, Darde ML, et al. Molecular approaches to diversity of populations of apicomplexan parasites. Int J Parasitol. 2009; 39(2):175-189.

2. Hill DE, Chirukandoth S, Dubey JP. Biology and epidemiology of Toxoplasma gondii in man and animals. Anim Health Res Rev. 2005; 6(1):41-61.

3. Black MW, Boothroyd JC. Lytic cycle of Toxoplasma gondii. Microbiol Mol Biol Rev. 2000;64(3):607-623.

4. Kamau ET, Srinivasan AR, Brown MJ, Fair MG, Caraher EJ, Boyle JP. A focused small-molecule screen identifies 14 compounds with distinct effects on Toxoplasma gondii. Antimicrob Agents Chemother. 2012; 56(11):5581-5590.

5. Boothroyd JC, Dubremetz JF. Kiss and spit: the dual roles of Toxoplasma rhoptries. Nat Rev Microbiol. 2008;6(1):79-88.

6. Curtis A, Wilkinson C. Nantotechniques and approaches in biotechnology. Trends Biotechnol. 2001;9(3):97-101.

7. Debbage P. Targeted drugs and nanomedicine: present and future. Curr Pharm Des. 2009;15(2):153-172.

8. Allahverdiyev AM, Abamor ES, Bagirova M, et al. Anti-leishmanial effect of silver nanoparticles and their enhanced antiparasitic activity under ultraviolet light. Int J Nanomedicine. 2011;6:2705-2714.

9. Adeyemi OS, Whiteley CG. Interaction of nanoparticles with arginine kinase from Trypanosoma brucei: kinetic and mechanistic evaluation. Int J Biol Macromol. 2013;62:450-456.

10. Bhardwaj R, Saudagar P, Dubey VK. Nanobiosciences: a contemporary approach in antiparasitic drugs. Mol Cell Pharmacol. 2012;4(3): 97-103. 
11. Butkus MA, Labare MP, Starke JA, Moon K, Talbot M. Use of aqueous silver to enhance inactivation of coliphage MS-2 by UV disinfection. Appl Environ Microbiol. 2004;70(5):2848-2853.

12. Adeyemi OS, Faniyan T. Antioxidant status in rats orally administered silver nanoparticle. J Taibah Univ Med Sci. 2014;9(3):182-186.

13. Adeyemi OS, Sulaiman FA. Evaluation of metal nanoparticles for drug delivery systems. J Biomed Res. 2015;29(2):145-149.

14. MubarakAli D, Thajuddin N, Jeganathan K, Gunasekaran M. Plant extract mediated synthesis of silver and gold nanoparticles and its antibacterial activity against clinically isolated pathogens. Colloids Surf B Biointerfaces. 2011;85(2):360-365.

15. Lakshmi JV, Sharath R, Chandraprabha MN, et al. Synthesis, characterization and evaluation of antimicrobial activity of zinc oxide nanoparticles. J Biochem Technol. 2012;3(5):S151-S154.

16. Das S, Bhattacharya A, Debnath N, Datta A, Goswami A. Nanoparticle-induced morphological transition of Bombyx mori nucleopolyhedrovirus: a novel method to treat silkworm grasserie disease. Appl Microbiol Biotechnol. 2013;97(13):6019-6030.

17. Rahul $\mathrm{S}$, Chandrashekhar $\mathrm{P}, \mathrm{Hemant} \mathrm{B}$, et al. In vitro antiparasitic activity of microbial pigments and their combination with phytosynthesized metal nanoparticles. Parasitol Int. 2015;64(5):353-356.

18. Adeyemi OS, Whiteley CG. Interaction of nanoparticles with recombinant arginine kinase from Trypanosoma brucei: thermodynamic and spectrofluorimetric evaluation. Biochim Biophys Acta. 2014;1840(1): 701-706

19. Wigginton NS, de Titta A, Piccapietra F, et al. Binding of silver nanoparticles to bacterial proteins depends on surface modifications and inhibits enzymatic activity. Environ Sci Technol. 2010;44(6):2163-2168.

20. Salma AA, Amer HA, Shaemaa HA, et al. The effects of gold and silver nanoparticles on transaminase enzymes activities. Int J Chem Res. 2011;1(4):2249-2329.

21. Srivastava M, Singh S, Self WT. Exposure to silver nanoparticles inhibits selenoprotein synthesis and the activity of thioredoxin reductase. Environ Health Perspect. 2012;120(1):56-61.

22. Ishiwa A, Kobayashi K, Takemae H, et al. Effects of dextran sulfates on the acute infection and growth stages of Toxoplasma gondii. Parasitol Res. 2013;112(12):4169-4176.

23. Kieschnick H, Wakefield T, Narducci CA, Beckers C. Toxoplasma gondii attachment to host cells is regulated by a calmodulin-like domain protein kinase. J Biol Chem. 2001;276(15):12369-12377.

24. Sugi T, Kobayashi K, Takemae H, et al. Identification of mutations in TgMAPK1 of Toxoplasma gondii conferring resistance to 1NM-PP1. Int J Parasitol Drugs Drug Resist. 2013;3:93-101.
25. Warleta F, Quesaada CS, Campos M, et al. Hydroxytyrosol protects against oxidative DNA damage in human breast cancer cells. Nutrients. 2011;2011(3):839-857.

26. Baraccaa A, Sgarbib G, Solainib G, et al. Rhodamine 123 as a probe of mitochondrial membrane potential: evaluation of proton flux through F0 during ATP synthesis. Biochim Biophys Acta. 2003;1606: $137-146$.

27. Pfefferkorn ER, Borotz SE, Nothnagel RF. Toxoplasma gondii: characterization of a mutant resistant to sulfonamides. Exp Parasitol. 1992; 74(3):261-270.

28. Saad HA, Soliman MI, Azzam AM, Mostafa B. Antiparasitic activity of silver and copper oxide nanoparticles against Entamoeba histolytica and Cryptosporidium parvum cysts. J Egypt Soc Parasitol. 2015;45(3): 593-602.

29. Saini P, Saha SK, Roy P, Chowdhury P, Sinha Babu SP. Evidence of reactive oxygen species (ROS) mediated apoptosis in Setaria cervi induced by green silver nanoparticles from Acacia auriculiformis at a very low dose. Exp Parasitol. 2016;160:39-48.

30. Ahmad A, Syed F, Shah A, et al. Silver and gold nanoparticles from Sargentodoxa cuneata: synthesis, characterization and antileishmanial activity. RSC Adv. 2015;2015(5):73793-73806.

31. Leyke S, Köhler-Sokolowska W, Paulke B, et al. Effects of nanoparticles in cells infected by Toxoplasma gondii. e-Polymers. 2013;12(1): 647-663.

32. Carruthers V, Boothroyd JC. Pulling together: an integrated model of Toxoplasma cell invasion. Curr Opin Microbiol. 2007;10(1):83-89.

33. Yah CS, Simate GS. Nanoparticles as potential new generation broad spectrum antimicrobial agents. Daru. 2015;23:43.

34. Charvat RA, Arrizabalaga G. Oxidative stress generated during monensin treatment contributes to altered Toxoplasma gondii mitochondria function. Sci Rep. 2016;15(6):22997.

35. Herrera B, Alvarez AM, Sanchez A, et al. Reactive oxygen species (ROS) mediates the mitochondrial-dependent apoptosis induced by transforming growth factor $\beta$ in fetal hepatocytes. FASEB J. 2001; 15(3):741-751.

36. Kuznetsov AV, Margreiter R, Amberger A, Saks V, Grimm M. Changes in mitochondrial redox state, membrane potential and calcium precede mitochondrial dysfunction in doxorubicin-induced cell death. Biochim Biophys Acta. 2011;1813(6):1144-1152.

37. Suski JM, Lebiedzinska M, Bonora M, Pinton P, Duszynski J, Wieckowski MR. Relation between mitochondrial membrane potential and ROS formation. Methods Mol Biol. 2012;810:183-205.
International Journal of Nanomedicine

\section{Publish your work in this journal}

The International Journal of Nanomedicine is an international, peerreviewed journal focusing on the application of nanotechnology in diagnostics, therapeutics, and drug delivery systems throughout the biomedical field. This journal is indexed on PubMed Central, MedLine, CAS, SciSearch $®$, Current Contents $\AA /$ Clinical Medicine,

\section{Dovepress}

Journal Citation Reports/Science Edition, EMBase, Scopus and the Elsevier Bibliographic databases. The manuscript management system is completely online and includes a very quick and fair peer-review system, which is all easy to use. Visit http://www.dovepress.com/ testimonials.php to read real quotes from published authors. 